\title{
Matrix metalloproteinases: effectors of development and normal physiology
}

\author{
Thiennu H. $\mathrm{Vu}^{1,3}$ and Zena Werb ${ }^{2}$ \\ ${ }^{1}$ Department of Medicine and Lung Biology Center, and ${ }^{2}$ Department of Anatomy, University of California, San Francisco, \\ California 94143 USA
}

The matrix metalloproteinase (MMP) family of extracellular proteinases regulates development and physiologic events. Genetic analyses using transgenic mice that have gain and loss of function of MMPs or of their endogenous inhibitors, the TIMPs, and pharmacogenetic studies with chemical inhibitors have begun to elucidate the roles that they play. It is now clear that these enzymes are important for cell migration, invasion, proliferation, and apoptosis. They regulate many developmental processes, including branching morphogenesis, angiogenesis, wound healing, and extracellular matrix degradation.

The matrix metalloproteinases (MMPs) are a family of extracellular matrix (ECM)-degrading enzymes that shares common functional domains and activation mechanisms (Sternlicht et al. 2000). These are $\mathrm{Ca}^{2+}$ - and $\mathrm{Zn}^{2+}$-dependent endopeptidases that are active at neutral $\mathrm{pH}$. They are synthesized as secreted or transmembrane proenzymes and processed to an active form by the removal of an amino-terminal propeptide. The propeptide is thought to keep the enzyme in latent form by the interaction of a cysteine residue in this peptide with the zinc moiety in the enzyme active site. Disruption of this interaction triggers the cysteine switch mechanism and results in activation of the enzyme. MMPs can be activated by chaotropic agents or by cleavage of the propeptide by members of the MMP family or by other proteases. They are inhibited by a family of tissue inhibitors of metalloproteinases, the TIMPs. As a family, MMPs degrade most components of the ECM. There are now $>20$ members of the MMP family. There are several distinct subgroups based on preferential substrates or similar structural domains: Collagenases that are active against fibrillar collagen, gelatinases that have high activity against denatured collagens, stromelysins that degrade noncollagen components of the ECM, membranetype MMPs (MT-MMPs) that are transmembrane molecules, and other less characterized members (Fig. 1; Table 1).

Because MMPs can degrade ECM molecules, their main function has been presumed to be remodeling of

\footnotetext{
${ }^{3}$ Corresponding author.

E-MAIL thiennu@itsa.ucsf.edu; FAX (415) 206-4123.

Article and publication are at www.genesdev.org/cgi/doi/10.1101/ gad. 815400 .
}

the ECM. They are thought to play important roles during embryonic development, as ECM remodeling is a critical component of tissue growth and morphogenesis. In fact, the discovery of MMPs was based on the observation that during amphibian metamorphosis, a collagenolytic activity has to be present to digest the collagens in tadpole tails (Gross and Lapiere 1962). The activity of MMPs during embryonic development may extend to more than the removal of unwanted ECM molecules, however. It is now clear that MMPs not only remodel the ECM, but also influence many cellular functions. MMP activity may be required during development and normal physiology in several ways: (1) to degrade ECM molecules and allow cell migration; (2) to alter the ECM micro-environment and result in alteration in cellular behavior; (3) to modulate the activity of biologically active molecules by direct cleavage, release from bound stores, or the modulating of the activity of their inhibitors (Fig 2). During tissue morphogenesis any number of these activities may contribute to the role that each MMP plays in a developmental process. Although insights into the activities of MMPs have emerged from in vitro studies, genetic and pharmacogenetic studies now indicate that MMPs do have important influence on many cellular functions. Two general approaches have been employed to identify the roles of MMPs during mammalian development: (1) general or tissue-specific expression of a transgene encoding an MMP or an MMP inhibitor (TIMP), and (2) generating null mutations in an MMP gene or TIMP gene using targeted mutagenesis. These approaches have given insights into the roles of several MMPs in development and normal physiology. The range of developmental effects seen in these function perturbation studies suggests that these enzymes do indeed participate as essential effectors of developmental processes in vivo.

\section{MMPs and cell migration}

It is evident that the ECM presents a barrier to cell migration. Cells that adhere to the ECM have to change from an adhesive phenotype to a migratory phenotype before they can move. This change requires several components: activation of cytoskeletal motor function to 
A MMPs with minimal domain (MMP-7)

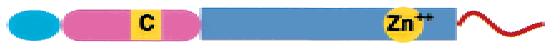

B MMPs with Hemopexin/Vitronectin domain

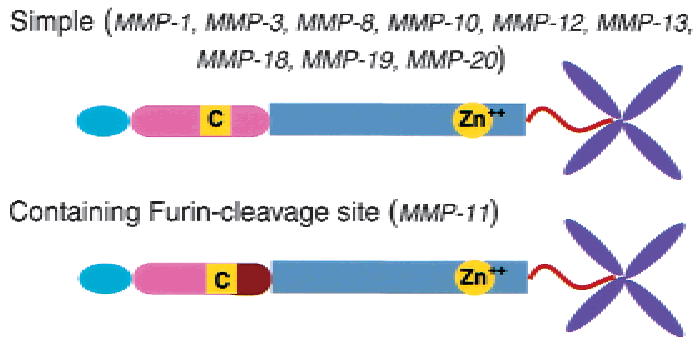

Transmembrane with Furin-cleavage site (MMP-14, MMP-15, MMP-16, MMP-17, MMP-24, MMP-25)

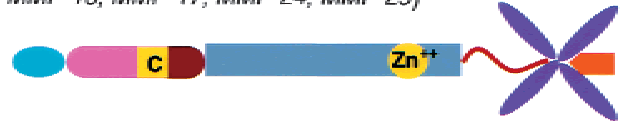

Containing Fibronectin type II repeat (MMP-2, MMP-9)
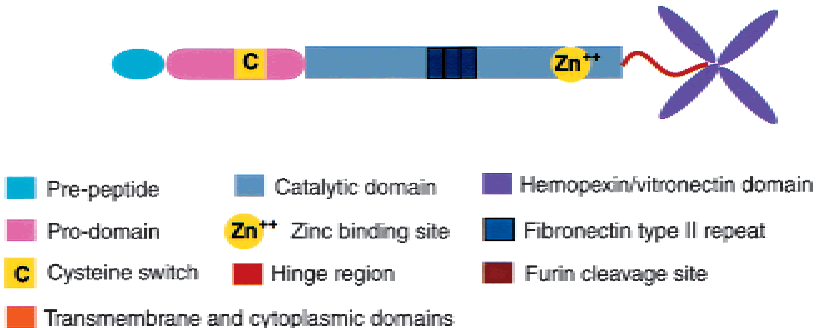

Figure 1. Structural domains of the matrix metalloproteinases (modified from Sternlicht et al. 2000). Diagrams of the domain structure of the different subgroups of the matrix metalloproteinase family. The prodomain contains a highly conserved sequence with an unpaired cysteine sulfhydryl group whose interaction with the active site zinc maintains the enzyme in latent form (the cysteine switch). The prodomain of some MMPs contains a recognition motif for furin-like enzymes, whose cleavage results in activation of the MMP. The catalytic domain of the gelatinases (MMP-2 and MMP-9) contains three gelatin-binding fibronectin type II repeats. The hemopexin/vitronectin domain is folded into a four-bladed propeller structure. (A) MMPs with minimal domain. (B) MMPs with hemopexin/ vitronectin domain.

provide cell movement, modulation of adhesive sites and cell-surface adhesive molecules to provide traction, clearing of ECM to break down physical barriers, and the presence of chemoattractants to guide migration. MMPs may potentially regulate any of these processes. Many in vitro studies have shown that MMPs can degrade a variety of ECM substrates, including collagens and noncollagenous molecules, suggesting that they can function as ECM-clearing enzymes during cell migration (Sternlicht et al. 2000). MMPs can also modulate the function of many biologically active molecules (see below) that participate in chemoattraction and migration. A role for MMPs during development is suggested by the necessity of cell migration, sometime over long distances, during tissue morphogenesis. This is evident as early as implan- tation in the extraembryonic tissues and during gastrulation in the embryos.

Studies using assays of cell migration through ECM barriers in culture have implicated MMPs in the migration of a variety of epithelial, mesenchymal, and neuronal cells either through the ECM or on specific ECM substrates. At the implantation site, trophoblasts must invade into the maternal decidua. These cells express high levels of MMP-9 (gelatinase B). In culture a neutralizing antibody against MMP-9 inhibits trophoblasts from invading and degrading ECM (Behrendtsen and Werb 1997). During angiogenesis, endothelial cells migrate into the surrounding ECM. In culture, the migration of endothelial cells through collagen or fibrin gels is impaired by MMP inhibitors (Fisher et al. 1994; Hiraoka et al. 1998). During bone remodeling, osteoclasts are recruited to the bone surfaces, and their migration may also depend on MMPs. In culture, MMP inhibitors curb the migration of purified osteoclasts through a collagen matrix (Sato et al. 1998). During the development of the nervous system, neurons may extend processes over long distances to form connections. In a model of neuronal differentiation, the neuroblastoma cell line SKSNBE expresses neuronal markers and establishes extensive neurite outgrowths in response to retinoic acid. MMP-9 is induced and found localized in the neurites, suggesting that it participates in neurite growth (Chambaut-Guérin et al. 2000). This is supported by another study showing that oligodendrocytes in culture expresses active metalloproteinases at the tip of their processes and that a neutralizing antibody against MMP-9 inhibits these outgrowths (Oh et al. 1999). Interestingly, oligodendrocytes from MMP-9-deficient mice fail to produce outgrowths in culture.

Epithelial morphogenesis depends on specific movements of epithelial cells. In culture, keratinocyte migration on collagen-1 requires specific cleavage of the collagen-1 molecule by a collagenase. MMP inhibitors curtail keratinocyte migration, and cells not expressing MMP-1 (collagenase-1) do not migrate on this substrate. In addition, keratinocytes do not migrate on a mutant type I collagen that lacks the collagenase cleavage site (Pilcher et al. 1997). In another system, breast epithelial cell migration on laminin-5 correlates with expression of MMP-14 (MT1-MMP) and MMP-2 (gelatinase A), and anti-sense oligodeoxynucleotides against MT1-MMP reduced their migration over this substrate (Koshikawa et al. 2000).

Organ cultures also show the requirement of MMPs in cell migration in systems that more closely approximate in vivo development. In early long bone development, (pre)osteoclastic cells invade into the cartilage anlage to initiate the process of endochondral ossification. These cells express high levels of MMP-9, and their migration into the cartilage matrix of skeletal elements in organ culture is limited by MMP inhibitors (Blavier and Delaissé 1995). In an explant culture model, MMP inhibitors dramatically alter mandibular morphogenesis (Chin and Werb 1997). In the absence of MMP activity, tongue development is severely affected. There is impaired mi- 
Table 1. The family of MMPs

\begin{tabular}{|c|c|c|c|}
\hline $\begin{array}{l}\text { MMP } \\
\text { designation }\end{array}$ & Name & Additional domains & $\begin{array}{l}\text { Furin- } \\
\text { activation } \\
\text { site }\end{array}$ \\
\hline MMP-1 & $\begin{array}{l}\text { collagenase-1, interstitial collagenase, } \\
\text { fibroblast collagenase }\end{array}$ & hinge, hemopexin/vitronectin & \\
\hline MMP-2 & gelatinase $\mathrm{A}, 72-\mathrm{kD}$ gelatinase & hinge, hemopexin, fibronectin repeats & \\
\hline MMP-3 & stromelysisn-1, transin-1 & extended hinge, hemopexin/vitronectin & \\
\hline MMP-7 & matrilysin, matrin, PUMP-1 & none & \\
\hline MMP-8 & neutrophil collagenase & hinge, hemopexin/vitronectin & \\
\hline MMP-9 & gelatinase $\mathrm{B}, 92-\mathrm{kD}$ gelatinase & hinge, hemopexin, fibronectin repeats & \\
\hline MMP-10 & stromelysin-2, transin-2 & extended hinge, hemopexin/vitronectin & \\
\hline MMP-11 & stromelysin-3 & hinge, hemopexin/vitronectin & yes \\
\hline MMP-12 & metalloelastase, macrophage elastase & hinge, hemopexin/vitronectin & \\
\hline MMP-13 & collagenase-3 & hinge, hemopexin/vitronectin & \\
\hline MMP-14 & MT1-MMP, membrane-type MMP & hinge, hemopexin/vitronectin, transmembrane & yes \\
\hline MMP-15 & MT2-MMP, MT-MMP-2 & hinge, hemopexin/vitronectin, transmembrane & yes \\
\hline MMP-16 & MT3-MMP, MT-MMP-3 & hinge, hemopexin/vitronectin, transmembrane & yes \\
\hline MMP-17 & MT4-MMP, MT-MMP-4 & hinge, hemopexin/vitronectin, transmembrane & yes \\
\hline MMP-18 & collagenase-4 (Xenopus) & hinge, hemopexin/vitronectin & \\
\hline MMP-19 & & hinge, hemopexin/vitronectin & \\
\hline MMP-20 & enamelysin & hinge, hemopexin/vitronectin & \\
\hline MMP-21 & & $\begin{array}{l}\text { no cysteine in propeptide, also has cysteine-rich, } \\
\text { proline-rich, IL-1R type II-like, transmembrane, and } \\
\text { cytoplasmic domains instead of hinge and hemopexin }\end{array}$ & yes \\
\hline MMP-22 & & same as MMP-21 & yes \\
\hline MMP-23 & & $\begin{array}{l}\text { short prodomain, short carboxy-terminal domain } \\
\text { with no sequence similarity to hemopexin }\end{array}$ & \\
\hline MMP-24 & MT5-MMP, MT-MMP-5 & hinge, hemopexin/vitronectin, transmembrane & yes \\
\hline MMP-25 & MT6-MMP, MT-MMP-6, leukolysin & hinge, hemopexin/vitronectin, transmembrane & yes \\
\hline
\end{tabular}

gration and proliferation of myoblasts into the tongue buds, the oral sulci fail to form, and fusion of the medial sulcus does not occur. Failure of the fusion of the medi- cal sulcus may be secondary to failure of epithelial cells to migrate away from the medial edges, and failure of the formation of the oral sulcus may result from impaired

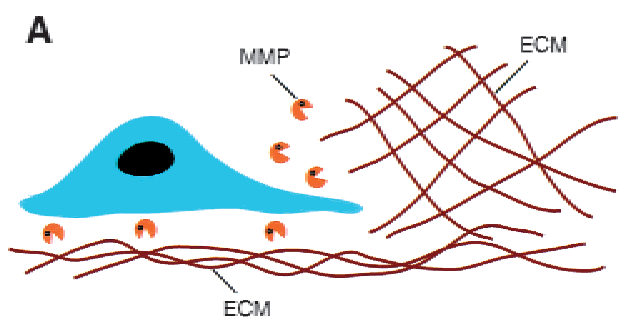

B

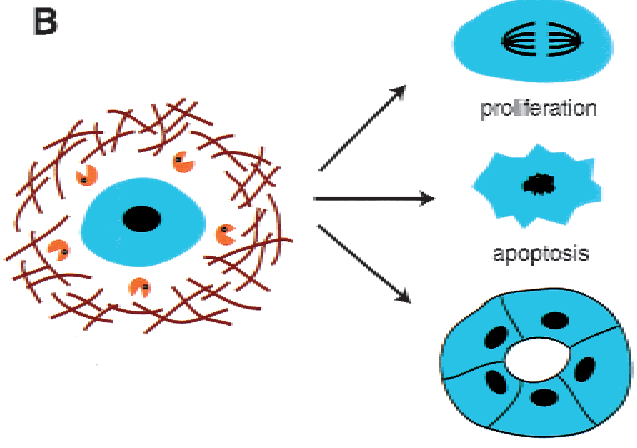

morphogenesis

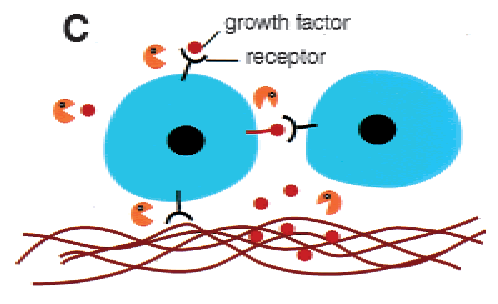

D

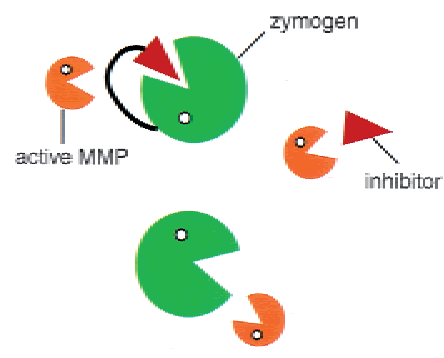

Figure 2. Modes of action of the matrix metalloproteinases. (A) MMPs may affect cell migration by changing the cells from an adhesive to nonadhesive phenotype and by degrading the ECM. $(B)$ MMPs may alter ECM microenvironment leading to cell proliferation, apoptosis, or morphogenesis. $(C)$ MMPs may modulate the activity of biologically active molecules such as growth factors or growth factor receptors by cleaving them or releasing them from the ECM. (D) MMPs may alter the balance of protease activity by cleaving the enzymes or their inhibitors. 
epithelial invagination, which in turn requires epithelial cell migration into the underlying matrix. In this model, there is a delay in the maturation and fusion of the component segments of Meckel's cartilage. This may be due to failure of the remodeling of the perichondrium that would allow cartilage elements to expand into the surrounding mesenchyme and to fuse. Interestingly, mandibles from mouse embryos lacking the EGF receptor express much lower amounts of MMPs and show an identical developmental defect in culture (Miettinen et al. 1999).

\section{ECM-dependent cell proliferation and apoptosis}

Interactions between ECM components and cell surface molecules regulate cell behavior. In a changing environment, the ability of cells to proliferate, survive, or differentiate changes as well. It is not surprising then that MMPs can alter cell behavior through their action on the ECM. Studies on the roles of MMPs on cell behavior in culture are few but have shown effects on cell proliferation, survival, apoptosis, differentiation, and organization. Inhibition of MMPs either by a chemical inhibitor or a neutralizing antibody to MMP-2 reduces the mitogenic response of cultured vascular smooth muscle cells to platelet derived growth factor (PDGF) (Uzui et al. 2000). Cultured airway smooth muscle cells also depends on MMP-2 for the proliferative response to a mitogen (Johnson and Knox 1999). These cells proliferate and secrete MMP-2 in response to treatment with either serum or PDGF, and an MMP inhibitor suppresses the proliferative response. Similarly, cultured human dermal microvascular endothelial cells (HDMEC) increase ECM deposition and suppress cell proliferation when MMP activities are inhibited (Kräling et al. 1999). In this case, MMP activity may be required to generate a proliferative signal. MMPs have also been shown to cause cell death. Proteinases or inappropriate ECM molecules induce apoptosis of mammary epithelial cells in culture presumably through altered signaling from integrins (Boudreau et al. 1996). ECM-degrading proteinases are induced in mammary cells in response to a fibronectin fragment (FN120), and inhibition of MMP activity rescues cell apoptosis (Schedin et al. 2000). In addition, MMP inhibitors also suppress smooth muscle cell proliferation and induce cell apoptosis in hypertrophied rat pulmonary arteries in organ culture (Cowan et al. 2000). Thus, MMPs affect cell survival and proliferation both positively and negatively by regulating survival signals generated by specific adhesive events. These differences in the effects of MMPs may reflect the differences in MMP substrates involved in each response.

\section{MMPs and tissue morphogenesis}

A particular action of MMPs of specific relevance to tissue morphogenesis is their ability to affect cell organization. When adipocytes are cultured on basement membrane, they migrate and organize into large multicellular clusters. These cells secrete MMP-2, and inhibition of MMP activity results in inhibition of cell migration and organization into three-dimensional structures (Brown et al. 1997). In an in vitro model of pancreatic islet development, embryonic pancreatic epithelial cells cultured in collagen gels differentiate and organize into clusters with characteristics of islets of Langerhans. Beta cells are located in the center and non-beta cells in the periphery. MMP-2 activity is induced, and inhibition of MMP activity by a chemical inhibitor abolishes islet morphogenesis without affecting endocrine cell differentiation (Miralles et al. 1998). Cells differentiate, but do not migrate and associate into islets.

Another ECM-dependent cell organization process is seen during tubular and branching morphogenesis, during angiogenesis, and during the development of many epithelial structures. Regulated and localized ECM remodeling may be required during tubular and branching morphogenesis because changes in cell proliferation, motility, shape, and formation of cellular contacts necessary for this process require precise interactions between cells and the ECM. Formation of tubular structures by endothelial and epithelial cells in three-dimensional culture shows varied dependence on MMPs. Tubule formation by HUVECs cultured on Matrigel or collagen gels is inhibited by inhibiting MMP activity (Schnaper et al. 1993; Fisher et al. 1994). Bovine aortic endothelial cells in collagen gels undergo apoptosis unless an angiogenic factor is present, in which case they survive, proliferate, and form capillary-like tubes. In this system, MMP inhibitor prevents apoptosis in the absence of an angiogenic factor and enhances tube formation in response to angiogenic factor (Kuzuya et al. 1999). Mammary epithelial cells in three-dimensional culture organize more into ductal structures than alveolar-like structures in response to a fibronectin fragment (FN120), and this process is dependent on MMP activity (Schedin et al. 2000). Overexpression of MMP-3 (stromelysin) in mammary epithelial cells induces epithelial-to-mesenchymal transition (Lochter et al. 1997; Sternlicht et al. 1999).

Organ cultures have been used extensively to show the role of MMPs in branching morphogenesis. When day 10 embryonic kidney explants are placed in culture, the ureteric bud develops by branching morphogenesis. This is inhibited by a neutralizing antibody against MMP-9 (Lelongt et al. 1997). MT1-MMP may also be involved in this process, as treatment of embryonic kidney cultures with anti-sense oligodeoxynucleotides against MT1MMP also decreases branching of the ureteric buds (Kanwar et al. 1999). In addition, anti-sense oligodeoxynucleotides against TIMP-2 increase branching and rescue the effect of anti-sense oligodeoxynucleotides against MT1-MMP.

In contrast to its effect in kidney development, inhibition of MMP activity in cultured salivary glands leads to increased branching (Fukuda et al. 1988). The difference in the effect of MMPs may be secondary to the mechanism of branching in the two epithelia. Kidney epithelium branches by budding and growing into the 
mesenchyme, requiring the degradation of the surrounding ECM. Salivary gland epithelium branches by formation and stabilization of clefts composed of collagen bundles in the epithelium concurrently with growth into the surrounding mesenchyme. Thus, in the kidney, ECM degradation would increase branching, but in the salivary glands enhanced ECM degradation would decrease branching, as it would lead to destabilization of the collagen clefts (Fig. 3).

Taken together these data suggest that MMPs play several distinct roles: altering ECM, permitting invasion, selection of branch points, regulating three-dimension organization, regulating epithelial-to-mesenchymal transition. Clearly these complex events suggest that multiple targets for MMP action must exist.

\section{MMPs and modulation of biologically active molecules}

A recurring paradigm in biologically active molecules is the generation of fragments with new activities by proteolytic cleavage. Several potent angiogenic inhibitors are produced by proteolytic cleavage: Angiogstatin is a fragment of plasminogen produced by cleavage in vitro by a number of MMPs, including MMP-3, MMP-7, MMP-9, and MMP-12 (macrophage metalloelastase) (O'Reilly et al. 1994; Dong et al. 1997; Patterson and Sang 1997; Lijnen et al. 1998), and in vivo by MMP-7 and MMP-9 (Pozzi et al. 2000). Mice that are deficient in the integrin alpha- 1 have decreased vascularization of tumor xenografts. This is due to increased level of circulating angiostatin generated by increased activity of MMP-7 and MMP-9 in these mice (Pozzi et al. 2000). Thus, increased expression of MMPs may both confer invasiveness to the tumor cells and, paradoxically, lead to production of molecules that limit their growth. Other angiogenic inhibitors generated by proteolysis have also been reported. Endostatin is a fragment of collagen XVIII with antiangiogenic activity (O'Reilly et al. 1997), and cleavage of antithrombin-III results in a molecule that is an inhibitor of angiogenesis (O'Reilly et al. 1999).

MMPs may also regulate the function of biologically active molecules by other mechanisms: They may control the molecules' bioavailability by releasing them from bound proteins or ECM stores, or they may regulate the molecules' activity by proteolytically activating or inactivating them. Thus, MMP activity can lead to both gain and loss of function of biologically active molecules. Examples for each of these mechanisms have been reported. MMP-1 and MMP-3 can degrade perlecan and release bound FGF (Whitelock et al. 1996). Cleavage of decorin by MMP-2, MMP-3, and MMP-7 (matrilysin) releases TGF- $\beta$ bound to decorin (Imai et al. 1997). MMPs may also regulate the bioavailability of growth factors by cleaving the proteins that bind to them. An example is the cleavage of insulin-like growth factorbinding proteins (IGFBPs) by MMPs. IGFBPs bind insulin-like growth factors (IGFs) with equal or higher affinity than IGF receptors, and degradation of IGFBPs is thought to play a major role in the regulation of IGF activity (Rajah et al. 1995). IGFBP-3 can be cleaved by
A

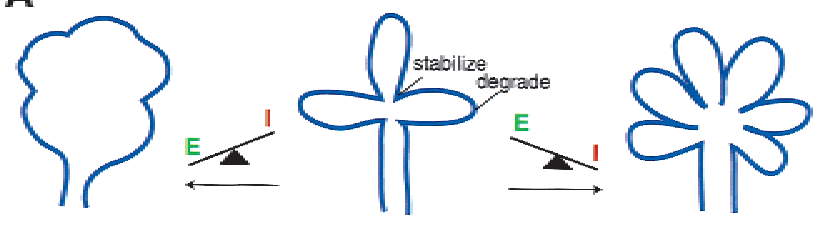

B

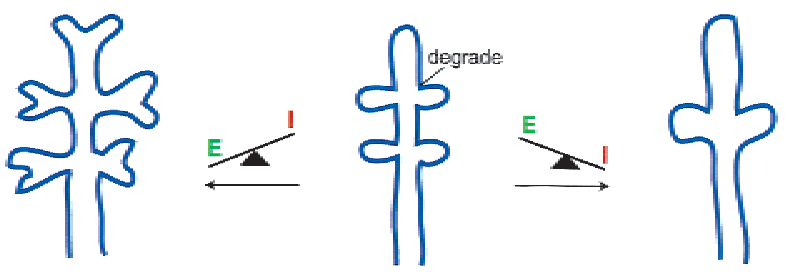

Figure 3. Epithelial branching morphogenesis depends on the balance of proteinases and proteinase inhibitors. (A) Submandibular gland epithelium branches by formation of stabilized collagen clefts concomitant with growth of the intervening epithelium. In this case, excess protease activity leads to less branching and excess inhibitor activity leads to more branching. (B) Kidney epithelium branches by budding of epithelium, which requires protease activity to grow into the surrounding mesenchyme. In this case, excess protease activity would lead to increased branching and excess inhibitor activity would lead to decreased branching.

MMP-1, MMP-2, and MMP-3, and IGFBP-5 is cleaved by MMP-1 and MMP-2 (Fowlkes et al. 1994a,b, 1995; Thrailkill et al. 1995; Wu et al. 1999). Of interest, mice overexpressing TIMP-1, an inhibitor of MMPs, have higher serum levels of IFGBP-3, lower levels of dissociable IGF-II, and decreased signaling through the IGF receptor (Martin et al. 1999). MMP activity is normally found in the liver, and liver of TIMP-1 overexpressing animals shows decreased proteolysis of IGFBP-3. MMP-2 and MMP-9 may also activate latent TGF- $\beta$ by cleaving its latent associated peptide (LAP; Yu and Stamenkovic 2000).

MMPs also affect the activity of other molecules through proteolytic cleavage. IL-1 $\beta$ precursor can be processed to its active form by MMP-2, MMP-3, and MMP-9 (Schönbeck et al. 1998). However, prolonged incubation with MMPs results in IL-1 $\beta$ degradation and loss of its biological activity (Ito et al. 1996). In some cases, cleavage of a molecule by the MMPs generates a more biologically active fragment. This is true for endothelin-1, which results in a peptide with greater vasoconstrictor effects when cleaved by MMP-2 (Fernandez-Patron et al. 1999). Another example is MMP-8 (neutrophil collagenase) cleavage of angiotensin-I, which generates angiotensin-II (Diekmann and Tschesche 1994). In other cases, although cleavage of a molecule by an MMP occurs, the resulting modulation of activity by such cleavage is not known, such as cleavage of substance P by MMP-9 and that of apolipoprotein (a) by MMP-12 (Backstrom and Tökés 1995; Edelstein et al. 1999). MMPs may also cleave cell surface molecules, thereby modulating their 
activity. An example is MMP-2 and MMP-9 cleavage of galectin-3 which alters the carbohydrate recognition domain of galectin-3 and reduces self-association of the galectin molecules. Thus, cleavage by MMPs may result in significant alteration in the signal-transducing property of this molecule (Ochieng et al. 1994, 1998).

MMPs may also modulate the activity of other proteinases. They may proteolytically activate latent proteases or inactivate their inhibitors. MMP-3 can cleave urokinase-type plasminogen activator (u-PA), separating the receptor-binding domain from its catalytic domain, without affecting catalytic activity (Orgel et al. 1998; Ugwu et al. 1998). The biological significance of this finding is unclear, but it is possible that removing receptor binding would result in decreased localization of u-PA to the cell surface. MMPs may also regulate the activity of other proteinases by degradation of their inhibitors. MMP-1, MMP-3, and MMP-9 can cleave $\alpha 1$ proteinase inhibitor and $\alpha 1$-antichymotrypsin (Mast et al. 1991). MMP-1, MMP-3, MMP-7, and MMP-9 proteolytically inactivate $\alpha 1$-proteinase inhibitor (Sires et al. 1994).

Even though it is clear from the above studies that MMPs can modulate the activity of a diverse number of biologically active molecules, there has been no direct evidence implicating this mechanism of MMP action in a developmental process. One may infer the role of MMPs in cases where the activity of a developmentally relevant molecule may be modulated by MMPs. For example, because FGF plays important roles in chondrocyte proliferation and differentiation, any modulation of its activity by MMPs by releasing it from ECM stores may be an important regulatory mechanism for cartilage development. Similarly, because TGF- $\beta$ has an important activity as a tubulogenic factor, the ability of MMPs to regulate its activity may be essential in branching morphogenesis.

The experiments described above have reached their conclusions by modulating MMP activity in culture. A drawback in these studies is that they have mainly used an inhibitor-based approach. Most of the inhibitors used are not completely specific to MMPs. The chemical inhibitors are broad-ranged. In addition the function discerned for an MMP in the isolated context of in vitro studies may not be its true function in the complex environment of the whole animal. Analysis of transgenic mice that have gain and loss of function of MMPs or their endogeneous inhibitors have given insights into the roles that these enzymes play in several developmental processes.

\section{MMP function during implantation}

Development of the placenta starts with the invasion and migration of trophoblast cells into the maternal tissue to establish connection with the maternal circulation (Rinkenberger et al. 1997). These trophoblasts express high levels of MMP-9 (Alexander et al. 1996a). In vitro MMP inhibition decreases migration and degradation of ECM by these cells. Evidence for in vivo role for
MMP-9 in implantation comes from studies of the Ets-2and MMP-9-null mice, as well as of TIMP-1 overexpressing mice and pharmacogenetic studies using chemical inhibitors. Ets-2 is a member of the Ets family of transcription factors that regulate the transcription of diverse genes, particularly MMPs. The Ets-2-deficient mice die early in embryogenesis, owing to defective development of the placenta (Yamamoto et al. 1998). The embryos die by embryonic day (E) 8.5. At E7.5, there is a smaller amount of attached trophoblastic tissue. At E6.5, the ectoplacental cone is small, apparently because of failure of trophoblast migration. A membrane, immunostained for laminin, a basement membrane component of Reichert's membrane, covers the ectoplacental cone and extends into and over the trophoblasts. There is also poor connection with the maternal circulation. Interestingly, in trophoblasts, expression of MMP-9, which is a transcriptional target of Ets-2, is significantly decreased in the Ets-2-null mice. Thus, the placental phenotype in Ets-2 deficiency may be due to insufficient MMP-9 activity in trophoblasts. In support of this model, TIMP-1 overexpressing, metalloproteinase inhibitor-treated mice (Alexander et al. 1996a) and MMP-9-null mice on some genetic backgrounds (J. Rinkenberger and Z. Werb, unpubl.) display similar placental phenotype, even though these do not lead to lethality. This severe lethal phenotype in the Ets-2 mice may be due to the effects of Ets-2 deficiency on other gene products. The mild phenotype of mice with MMP-9 deficiency compared with those overexpressing TIMP-1 or those treated with chemical inhibitors suggests that other MMPs and/or other metalloproteinases also contribute. Neither the chemical inhibitors nor the TIMPS are completely specific to MMPs. For example, TIMP-1 also inhibits aggrecanase, an ADAM-TS family member, and TIMP-3 also inhibits TACE, a shedding enzyme that is a member of the ADAM family and regulates availability of TGF- $\alpha$.

\section{MMPs and wound healing}

Healing of a skin wound requires several processes similar to development: cell migration, ECM degradation, and tissue reorganization (Fig. 4). Keratinocytes at the edge of the wound have to migrate to re-epithelialize the wound surface. Then the fibrin-rich provisional matrix that is laid down following wounding must be removed. The dermis also contributes by contracting to facilitate wound closure. Studies in culture have shown the need for MMP-1 activity in keratinocyte migration. In animal studies MMP activity is implicated in keratinocyte migration and dermal contraction. Wound healing is retarded in mice treated with an MMP inhibitor (Lund et al. 1999|, due to decreased keratinocyte migration. Interestingly, plasminogen-deficient mice also have a similar wound-healing defect, suggesting that both plasmin and MMPs are important for keratinocyte migration during wound healing (Romer et al. 1996). In addition, keratinocyte migration is completely inhibited in the plasminogen-deficient mice treated with an MMP inhibitor, showing that these two enzyme families act synergisti- 


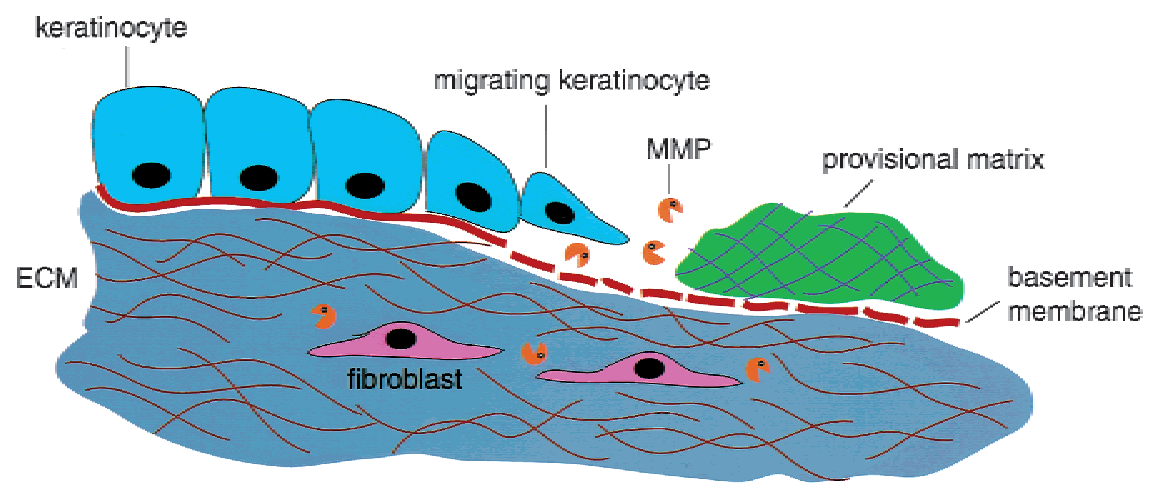

Figure 4. Dermal wound healing. At the wound edge, keratinocytes migrate into the denuded area to re-epithelialize the wound. These cells migrate on a collagen substrate. During this process, the provisional matrix consisting of fibrin and plasma proteins is removed. In the subcutaneous tissue, fibroblasts contract the ECM to facilitate wound closure. cally (Lund et al. 1999). As discussed above, it is difficult to implicate only MMPs in processes that are inhibited by a chemical inhibitor. These inhibitors are not entirely specific. Direct evidence for a role of MMPs in wound healing comes from studies of MMP-3-null mice. These studies showed that MMP-3 is important for wound contraction. The MMP-3-null mice fail to upregulate a multicellular contractile ring of actin in dermal fibroblasts and fail to contract the wound, and thus delay wound healing (Bullard et al. 1999a). Of interest, MMP-3-null dermal fibroblasts in culture contract collagen gels significantly less that wild-type fibroblasts, suggesting that MMP-3 expression by fibroblasts is important in wound contraction (Bullard et al. 1999b).

\section{MMPs in mammary development}

The mammary glands form during embryonic development by the budding of an epithelial tube into the mammary fat pad and subsequent extensive growth at the terminal end bud and branching to form the epithelial ducts (Henninghausen and Robinson 1998). The ducts end in terminal lobular-alveolar units that differentiate into alveoli. These alveoli are the milk-secreting glands during lactation. The alveoli develop during pregnancy and lactation and regress (involute) during weaning. Because of the significant amount of ECM remodeling that has to take place during virgin development, lactation, and weaning, MMPs would be expected to play a pivotal role in mammary branching morphogenesis.

Targeted expression of an autoactivating isoform of stromelysin-1 to the mammary gland alters development of the gland (Sympson et al. 1994; Witty et al. 1995). Virgin female mice, in whom there is low level of the transgene expression in mammary epithelia, show abnormal morphology of the gland. The primary ducts have supernumerary branches and the alveoli show precocious development, with $\beta$-casein expression comparable to that seen in glands at early to middle pregnancy. Lactating glands have high levels of transgene expression and show loss of basement membrane integrity, with loss of laminin and collagen IV. This results in alveoli with decreased size and shrunken lumen. During late pregnancy, the alveolar epithelial cells in the transgenic mice undergo unscheduled apoptosis. Interestingly, there is enhanced cleavage of the basement membrane molecule entactin (nidogen-1) around apoptotic cells, which parallels extracellular MMP activity. When stromelysin-1 transgenic mice are crossed with mice overexpressing TIMP-1 the unscheduled apoptosis of epithelial cells is extinguished (Alexander et al. 1996b). These studies show that the inappropriate expression of active stromelysin-1 can lead to altered tissue morphogenesis. Its mechanism of action is not clear, but may include alteration in epithelial cell proliferation and morphogenesis, leading to increased ductal branching, as well as regulation of alveolar epithelial cell differentiation and apoptosis. Indeed, increased ductal branching has also been observed in mice with a transgene expressing anti-sense RNA against TIMP-1 targeted to the mammary gland (Fata et al. 1999). Epithelial cells in these ducts showed increased proliferation. In contrast, implantation of TIMP-1-releasing pellets in the mammary glands results in attenuated ductal expansion and decreased proliferation of epithelium in terminal end buds in the vicinity of the pellets. TIMP-1-overexpressing mice also show a decrease in ductal invasion into the mammary fat pads (M. Sciabica and Z. Werb, unpubl.).

It may be argued that overexpression of MMPs or TIMPs may lead to a response that is not physiologic. Studies of the effects of the gain and loss of function of TIMP-1 also have the problem of specificity. As discussed above, TIMP-1 also inhibits metalloproteinases other than MMPs. The only direct evidence implicating MMPs in mammary gland development comes for studies of the stromelysin-1-deficient mice. These mice have a mild abnormality in mammary gland development, but the defect is not pronounced. There is a small decrease in branching morphogenesis of the ducts, with fewer secondary and tertiary branching. These data indicate that other MMPs may have the major responsibility for ductal morphogenesis. From expression data, the most likely candidates are MMP-2 and MT1-MMP.

\section{MMPs in bone development}

The skeleton develops by one of two processes: intramembranous or endochondral ossification (Caplan 1988; Karsenty 1999|. The long bones of the axial skeleton and 
the spine form by endochondrial ossification, in which a cartilage template is laid down through an orderly process of chondrocyte proliferation and maturation to form hypertrophic cartilage. Hypertrophic cartilage is subsequently resorbed and replaced by bone matrix following the invasion of capillaries. The resorption of hypertrophic cartilage ECM appears to take place through the action of chondroclasts, cells that invade into the cartilage preceding capillaries. Following capillary invasion, osteoblasts are recruited to form the trabecular bones, which are subsequently remodeled by osteoclasts. Osteoblasts and osteoclasts maintain bone homeostasis in the adults.

The flat bones of the skull form by intramembranous ossification, whereby osteoblasts differentiate and deposit bone matrix onto a cartilage primordium, which is subsequently removed. The bone matrix is also vascularized and remodeled by osteoclasts, but how this occurs is not clear. Thus, the formation of bones requires active remodeling of specialized matrices /cartilage and bone) as well as coordinated activities of many cell types (chondrocytes, endothelial cells, chondroclasts, osteoblasts, osteoclasts, and perhaps others). Recent gene targeting studies have implicated two particular MMPs in bone development: MMP-9 and MT1-MMP.

Targeted inactivation of the MMP-9 gene resulted in a very specific defect in endochondral bone formation (Vu et al. 1998). Mice with this defect accumulate hypertrophic cartilage at the skeletal growth plates. This is not due to abnormality in proliferation and maturation of chondrocytes, but is secondary to impaired endochondral ossification. Vascular invasion into the hypertrophic cartilage zone is impeded. This is coupled with a delay in terminal hypertrophic chondrocyte apoptosis, showing that hypertrophic chondrocytes do not have a fixed life span and automatically undergo apoptosis, but their death is coupled with capillary invasion. Only those hypertrophic chondrocytes in proximity to the capillaries undergo cell death. There are several models for the function of MMP-9 in endochondral bone formation: resorption of cartilage, regulation of the availability of an angiogenic factor, or regulation of hypertrophic chondrocyte apoptosis. MMP-9-null hypertrophic cartilage in culture has a decrease in net release of an angiogenic activity. The simplest model for the action of MMP-9 in this process is that of regulating the release from hypertrophic cartilage ECM stores of an angiogenic factor. Thus, lack of MMP-9 leads to a decrease in vascular invasion with its attendant processes. Alternatively, MMP-9 may have other functions and the decrease in angiogenic activity of MMP-9-null hypertrophic cartilage may be an indirect consequence.

The relevant angiogenic factor at the growth plate is VEGF, which is expressed by hypertrophic cartilage. Inhibition of VEGF activity by a soluble VEGF receptor, mFlt-IgG, leads to inhibition of vascular ingrowth into hypertrophic cartilage (Gerber et al. 1999). The resulting accumulation of hypertrophic cartilage is similar to, but more severe than, that seen in the MMP-9-null animals. However, VEGF appears to do more than regulating an- giogenesis at the growth plate. Treatment with mFlt-IgG also leads to decreased recruitment of chondroclasts and osteoblasts. Thus, VEGF seems to be a key regulator of the diverse processes required for organized and coordinated endochondral ossification. MMPs may participate in this control by directly or indirectly regulating the net balance of VEGF activity. The defect in endochondral ossification is the only noticeable developmental defect in these mice, pointing to a very specialized function of MMP-9 in development.

Targeted inactivation of MT1-MMP, a transmembrane MMP, results in several skeletal defects that are different from that in the MMP-9-null mice (Holmbeck et al. 1999). The MT1-MMP deficient mice have craniofacial dysmorphisms caused by impaired intramembraneous bone formation and dwarfism that is most likely secondary to impaired endochondral ossification. Surprisingly, they also have osteopenia, arthritis, and fibrosis of the soft tissues. Thus, too little MMP can lead to tissue destruction, a role hypothesized for too much MMP. The abnormal cranial morphogenesis is partly due to impaired removal of the cavarial cartilage primordia, which persist and transform into a fibrotic vestige. This may interfere with the normal formation of the calvarial bones and suture closure. In the long bones, there is defective ossification of the epiphysis. In the epiphysis, hypertrophic cartilage is formed in the center from the inward maturation of chondrocytes. This hypertrophic cartilage is then ossified by the invasion into the cartilage of perichondrial vessels through vascular canals formed from the perichondrium. In the MT1-MMP-deficient mice, no vascular canal is formed. Therefore, ossification is delayed and only occurs when the hypertrophic cartilage zone has expanded to perichondrium, by the invasion of vessels directly from the perichondrium. Following this delayed ossification of the epiphysis, the growth plates become disorganized with decreased proliferation of chondrocytes. This may lead to the dwarfism seen in these mice. Progressive fibrosis develops in periskeletal soft tissues in these mice, including the periosteum, tendon, ligament, and capsular insertions. These may limit bone growth leading to dwarfism. Similar defects have been observed in mice with a mutation in the collagenase cleavage site of type I collagen (Liu et al. 1995). Bone marrow stromal cells and fibroblasts isolated from the MT1-MMP-null mice have impaired collagenolytic activity in an in vitro assay, suggesting that the developmental defects in these mice may result from an impairment in the remodeling of the skeletal matrix as well as of the periskeletal soft tissues. Of interest is the finding that there is decreased osteogenesis from MT1-MMP bone marrow stromal cells, suggesting that the differentiation or function of osteoblasts may also be impaired. In addition, there is increased osteoclast number in these mice, indicating that their differentiation and recruitment may also be affected in MT1-MMP deficiency. This effect of MT1-MMP may not be secondary to its collagenolytic activity but could conceivably be due to its activity on growth or differentiation factors for these cells. This remains to be shown. 


\section{Conclusions}

Discovering the roles of the MMPs in physiological processes has not been simple. In vitro studies suggest that MMPs can affect fundamental cellular processes such as proliferation, survival, migration, and morphogenesis. These are the key processes of development. Yet, except for the ones discussed above, developmental defects resulting from inactivation of other MMP genes have not been apparent. In addition, many processes that are affected in vitro by alteration of MMP activity do not seem to be affected by the lack of the relevant MMP in vivo. For example, in vitro kidney development shows a dependency on MMP-9 and MT1-MMP. Yet, kidney development is not greatly affected in either the MMP-9 or MT1-MMP knockout mice. It is possible that there are compensatory mechanisms in vivo that are not available in the in vitro system. Inactivating individual MMPs may show defects in processes in which the particular MMP play an indispensable function, but may not reveal minor functions. In addition, it is usually difficult to decipher the mechanisms of action of the relevant MMP from looking at the end result of its deficiency in what is usually a very complex interplay of many cellular processes.

Another fundamental question in the function of MMPs is that of the relevant in vivo substrates of MMPs. It is clear that fundamentally these enzymes exert their effects by degrading either ECM or other molecules, but what these are in each specific case has eluded discovery. Even though we have gained significant insights into how MMPs may function, more work needs to be done before the roles of MMPs in development are fully elucidated. Inactivation of other MMPs, inactivation of multiple MMPs, and discovering the relevant substrates will be the focus of future studies. As more data are generated, the pieces may eventually come together.

\section{Acknowledgments}

This work was supported by grants from the National Institutes of Health (CA57621, CA72006, AR46238, DE13058), by a grant form the Human Frontiers Science Program (RG0051/1999M), and by a Mentored Physician-Scientist Award (K08 HL03880).

\section{References}

Alexander, C.M., Hansell, E.J., Behrendtsen, O., Flannery, M.L., Kishnani, N.S., Hawkes, S.P., and Werb, Z. 1996a. Expression and function of matrix metalloproteinases and their inhibitors at the maternal-embryonic boundary during mouse embryo implantation. Development 122: 1723-1736.

Alexander, C.M., Howard, E.W., Bissell, M.J., and Werb, Z. 1996b. Rescue of mammary epithelial cell apoptosis and entactin degradation by a tissue inhibitor of metalloproteinases-1 transgene. J. Cell Biol. 135: 1669-1677.

Backstrom, J.R. and Tökés, Z.A. 1995. The 84-kDa form of human matrix metalloproteinase- 9 degrades substance $P$ and gelatin. J. Neurochem. 64: 1312-1318.

Behrendtsen, O. and Werb, Z. 1997. Metalloproteinases regulate parietal endoderm differentiating and migrating in cultured mouse embryos. Develop. Dyn. 208: 255-265.

Blavier, L. and Delaissé, J.M. 1995. Matrix metalloproteinases are obligatory for the migration of preosteoclasts to the developing marrow cavity of primitive long bones. J. Cell Sci. 108: $3649-3659$.

Boudreau, N., Werb, Z., and Bissell, M.J. 1996. Suppression of apoptosis by basement membrane requires three-dimensional tissue organization and withdrawal from the cell cycle. Proc. Natl. Acad. Sci. 93: 3509-3513.

Brown, L.M., Fox, H.L., Hazen, S.A., LaNoue, K.F., Rannels, S.R., and Lynch, C.J. 1997. Role of the matrixin MMP-2 in multicellular organization of adipocytes cultured in basement membrane components. Am. J. Physiol. 272: C937C949.

Bullard, K.M., Lund, L., Mudgett, J.S., Mellin, T.N., Hunt, T.K., Murphy, B., Ronan, J., Werb, Z., and Banda, M.J. 1999a. Impaired wound contraction in stromelysin-1-deficient mice. Ann. Sur. 230: 260-265.

Bullard, K.M., Mudgett, J., Scheuenstuhl, H., Hunt, T.K., and Banda, M.J. 1999b. Stromelysin-1-deficient fibroblasts display impaired contraction in vitro. J. Surg. Res. 84: 31-34.

Caplan, A.I. 1988. Bone development. Ciba Found. Symp. 136: 3-21.

Chambaut-Guérin, A.M., Hérigault, S., Rouet-Benzineb, P., Rouher, C., and Lafuma, C. 2000. Induction of matrix metalloproteinase MMP-9 (92-kDa gelatinase) by retinoic acid in human neuroblastoma SKNBE cells: Relevance to neuronal differentiation. J. Neurochem. 74: 508-517.

Chin, J.R. and Werb, Z. 1997. Matrix metalloproteinases regulate morphogenesis, migration and remodeling of epithelium, tongue skeletal muscle and cartilage in the mandibular arch. Development 124: 1519-1530.

Cowan, K.N., Jones, P.L., and Rabinovitch, M. 2000. Elastase and matrix metalloproteinase inhibitors induce regression, and tenascin- $\mathrm{C}$ antisense prevents progression, of vascular disease. J. Clin. Invest. 105: 21-34.

Diekmann, O. and Tschesche, H. 1994. Degradation of kinins, angiotensins and substance $\mathrm{P}$ by polymorphonuclear matrix metalloproteinases MMP 8 and MMP 9. Brazil. J. Med. Biol. Res. 27: 1865-1876.

Dong, Z., Kumar, R., Yang, X., and Fidler, I.J. 1997. Macrophagederived metalloelastase is responsible for the generation of angiostatin in Lewis lung carcinoma. Cell 88: 801-810.

Edelstein, C., Shapiro, S.D., Klezovitch, O., and Scanu, A.M. 1999. Macrophage metalloelastase, MMP-12, cleaves human apolipoprotein(a) in the linker region between kringles IV-4 and IV-5. Potential relevance to lipoprotein(a) biology. J. Biol. Chem. 274: 10019-10023.

Fata, J.E., Leco, K.J., Moorehead, R.A., Martin, D.C., and Khokha, R. 1999. Timp-1 is important for epithelial proliferation and branching morphogenesis during mouse mammary development. Develop. Biol. 211: 238-254.

Fernandez-Patron, C., Radomski, M.W., and Davidge, S.T. 1999. Vascular matrix metalloproteinase-2 cleaves big endothelin-1 yielding a novel vasoconstrictor. Circ. Res. 85: 906911.

Fisher, C., Gilbertson-Beadling, S., Powers, E.A., Petzold, G., Poorman, R., and Mitchell, M.A. 1994. Interstitial collagenase is required for angiogenesis in vitro. Develop. Biol. 162: 499-510.

Fowlkes, J.L., Enghild, J.J., Suzuki, K., and Nagase, H. 1994a. Matrix metalloproteinases degrade insulin-like growth factor-binding protein-3 in dermal fibroblast cultures. J. Biol. Chem. 269: 25742-25746.

Fowlkes, J.L., Suzuki, K., Nagase, H., and Thrailkill, K.M. 
1994b. Proteolysis of insulin-like growth factor binding protein-3 during rat pregnancy: A role for matrix metalloproteinases. Endocrinology 135: 2810-2813.

Fowlkes, J.L., Thrailkill, K.M., Serra, D.M., Suzuki, K., and Nagase, H. 1995. Matrix metalloproteinases as insulin-like growth factor binding protein-degrading proteinases. Prog. Growth Factor Res. 6: 255-263.

Fukuda, Y., Masuda, Y., Kishi, J., Hashimoto, Y., Hayakawa, T., Nogawa, H., and Nakanishi, Y. 1988. The role of interstitial collagens in cleft formation of mouse embryonic submandibular gland during initial branching. Development 103: 259-267.

Gerber, H.P., Vu, T.H., Ryan, A.M., Kowalski, J., Werb, Z., and Ferrara, N. 1999. VEGF couples hypertrophic cartilage remodeling, ossification and angiogenesis during endochondral bone formation. Nat. Med. 5: 623-628.

Gross, J. and Lapiere, C.M. 1962. Collagenolytic activity in amphibian tissues: A tissue culture assay. Proc. Natl. Acad. Sci. 48: 1014-1022.

Henninghausen, L. and Robinson, G.W. 1998. Think globally, act locally: The making of a mouse mammary gland. Genes \& Dev. 12: 449-455.

Hiraoka, N., Allen, E., Apel, I.J., Gyetko, M.R., and Weiss, S.J. 1998. Matrix metalloproteinases regulate neovascularization by acting as pericellular fibrinolysins. Cell 95: 365-377.

Holmbeck, K., Bianco, P., Caterina, J., Yamada, S., Kromer, M., Kuznetsov, S.A., Mankani, M., Robey, P.G., Poole, A.R., Pidoux, I., Ward, J.M., and Birkedal-Hansen, H. 1999. MT1MMP-deficient mice develop dwarfism, osteopenia, arthritis, and connective tissue disease due to inadequate collagen turnover. Cell 99: 81-92.

Imai, K., Hiramatsu, A., Fukushima, D., Pierschbacher, M.D., and Okada, Y. 1997. Degradation of decorin by matrix metalloproteinases: Identification of the cleavage sites, kinetic analyses and transforming growth factor-betal release. Biochem. J. 322: 809-814.

Ito, A., Mukaiyama, A., Itoh, Y., Nagase, H., Thogersen, I.B., Enghild, J.J., Sasaguri, Y., and Mori, Y. 1996. Degradation of interleukin $1 \beta$ by matrix metalloproteinases. J. Biol. Chem. 271: $14657-14660$.

Johnson, S. and Knox, A. 1999. Autocrine production of matrix metalloproteinase-2 is required for human airway smooth muscle proliferation. Am. J. Physiol. 277: L1109-L1117.

Kanwar, Y.S., Ota, K., Yang, Q., Wada, J., Kashihara, N., Tian, Y., and Wallner, E.I. 1999. Role of membrane-type matrix metalloproteinase 1 (MT-1-MMP), MMP-2, and its inhibitor in nephrogenesis. Am. J. Physiol. 277: F934-F947.

Karsenty, G. 1999. The genetic transformation of bone biology. Genes \& Dev. 13: 3037-3051.

Koshikawa, N., Giannelli, G., Cirulli, V., Miyazaki, K., and Quaranta, V. 2000. Role of cell surface metalloprotease MT1-MMP in epithelial cell migration over laminin-5. J. Cell Biol. 148: 615-624.

Kräling, B.M., Wiederschain, D.G., Boehm, T., Rehn, M., Mulliken, J.B., and Moses, M.A. 1999. The role of matrix metalloproteinase activity in the maturation of human capillary endothelial cells in vitro. J. Cell Sci. 112: 1599-1609.

Kuzuya, M., Satake, S., Ramos, M.A., Kanda, S., Koike, T., Yoshino, K., Ikeda, S., and Iguchi, A. 1999. Induction of apoptotic cell death in vascular endothelial cells cultured in three-dimensional collagen lattice. Exp. Cell Res. 248: 498508 .

Lelongt, B., Trugnan, G., Murphy, G., and Ronco, P.M. 1997. Matrix metalloproteinases MMP2 and MMP9 are produced in early stages of kidney morphogenesis but only MMP9 is required for renal organogenesis in vitro. J. Cell Biol.
136: $1363-1373$.

Lijnen, H.R., Ugwu, F., Bini, A., and Collen, D. 1998. Generation of an angiostatin-like fragment from plasminogen by stromelysin-1 (MMP-3). Biochemistry 37: 4699-4702.

Liu, X., Wu, H., Byrne, M., Jeffrey, J., Krane, S., and Jaenisch, R. 1995. A targeted mutation at the known collagenase cleavage site in mouse type I collagen impairs tissue remodeling. I. Cell Biol. 130: 227-237.

Lochter, A., Galosy, S., Muschler, J., Freedman, N., Werb, Z., and Bissell, M.J. 1997. Matrix metalloproteinase stromelysin-1 triggers a cascade of molecular alterations that leads to stable epithelial-to-mesenchymal conversion and a premalignant phenotype in mammary epithelial cells. J. Cell Biol. 139: 1861-1872.

Lund, L.R., Romer, J., Bugge, T.H., Nielsen, B.S., Frandsen, T.L., Degen, J.L., Stephens, R.W., and Danø, K. 1999. Functional overlap between two classes of matrix-degrading proteases in wound healing. EMBO I. 18: 4645-4656.

Martin, D.C., Fowlkes, J.L., Babic, B., and Khokha, R. 1999. Insulin-like growth factor II signaling in neoplastic proliferation is blocked by transgenic expression of the metalloproteinase inhibitor TIMP-1. J. Cell Biol. 146: 881-892.

Mast, A.E., Enghild, J.J., Nagase, H., Suzuki, K., Pizzo, S.V., and Salvesen, G. 1991. Kinetics and physiologic relevance of the inactivation of alpha 1-proteinase inhibitor, alpha 1-antichymotrypsin, and antithrombin III by matrix metalloproteinases-1 (tissue collagenase), -2 (72-kDa gelatinase/type IV collagenase), and -3 (stromelysin). J. Biol. Chem. 266: 1581015816.

Miettinen, P.J., Chin, J.R., Shum, L., Slavkin, H.C., Shuler, C.F., Derynck, R., and Werb, Z. 1999. Epidermal growth factor receptor function is necessary for normal craniofacial development and palate closure. Nat. Genet. 22: 69-73.

Miralles, F., Battelino, T., Czernichow, P., and Scharfmann, R. 1998. TGF-beta plays a key role in morphogenesis of the pancreatic islets of Langerhans by controlling the activity of the matrix metalloproteinase MMP-2. J. Cell Biol. 143: 827836.

Ochieng, J., Fridman, R., Nangia-Makker, P., Kleiner, D.E., Liotta, L.A., Stetler-Stevenson, W.G., and Raz, A. 1994. Galectin-3 is a novel substrate for human matrix metalloproteinases-2 and -9. Biochemistry 33: 14109-14114.

Ochieng, J., Green, B., Evans, S., James, O., and Warfield, P. 1998. Modulation of the biological functions of galectin-3 by matrix metalloproteinases. Biochim. Biophys. Acta 1379: 97-106.

Oh, L.Y., Larsen, P.H., Krekoski, C.A., Edwards, D.R., Donovan, F., Werb, Z., and Yong, V.W. 1999. Matrix metalloproteinase-9/gelatinase B is required for process outgrowth by oligodendrocytes. J. Neurosci. 19: 8464-8475.

O'Reilly, M.S., Holmgren, L., Shing, Y., Chen, C., Rosenthal, R.A., Moses, M., Lane, W.S., Cao, Y., Sage, E.H., and Folkman, J. 1994. Angiostatin: A novel angiogenesis inhibitor that mediates the suppression of metastases by a Lewis lung carcinoma. Cell 79: 315-328.

O'Reilly, M.S., Boehm, T., Shing, Y., Fukai, N., Vasios, G., Lane, W.S., Flynn, E., Birkhead, J.R., Olsen, B.R., and Folkman, J. 1997. Endostatin: An endogenous inhibitor of angiogenesis and tumor growth. Cell 88: 277-285.

O'Reilly, M.S., Pirie-Shepherd, S., Lane, W.S., and Folkman, J. 1999. Antiangiogenic activity of the cleaved conformation of the serpin antithrombin. Science 285: 1926-1928.

Orgel, D., Schröder, W., Hecker-Kia, A., Weithmann, K.U., Kolkenbrock, H., and Ulbrich, N. 1998. The cleavage of prourokinase type plasminogen activator by stromelysin-1. Clin. Chem. Lab. Med. 36: 697-702. 
Patterson, B.C. and Sang, Q.A. 1997. Angiostatin-converting enzyme activities of human matrilysin (MMP-7) and gelatinase B/type IV collagenase (MMP-9). J. Biol. Chem. 272: 2882328825.

Pilcher, B.K., Dumin, J.A., Sudbeck, B.D., Krane, S.M., Welgus, H.G., and Parks, W.C. 1997. The activity of collagenase-1 is required for keratinocyte migration on a type I collagen matrix. J. Cell Biol. 137: 1445-1457.

Pozzi, A., Moberg, P.E., Miles, L.A., Wagner, S., Soloway, P., and Gardner, H.A. 2000. Elevated matrix metalloproteases and angiostatin levels in integrin alpha 1 knockout mice cause reduced tumor vasularization. Proc. Nat1. Acad. Sci. 97: 2202-2207.

Rajah, R., Katz, L., Nunn, S., Solberg, P., Beers, T., and Cohen, P. 1995. Insulin-like growth factor binding protein (IGFBP) proteases: Functional regulators of cell growth. Prog. Growth Factor Res. 6: 273-284.

Rinkenberger, J.L., Cross, J.C., and Werb, Z. 1997. Molecular genetics of implantation in the mouse. Develop. Genet. 21: $6-20$.

Romer, J., Bugge, T.H., Pyke, C., Lund, L.R., Flick, M.J., Degen, J.L., and Dano, K. 1996. Impaired wound healing in mice with a disrupted plasminogen gene. Nat. Med. 2: 287-292.

Sato, T., Foged, N.T., and Delaissé, J.M. 1998. The migration of purified osteoclasts through collagen is inhibited by matrix metalloproteinase inhibitors. J. Bone Mineral Res. 13: 5966.

Schedin, P., Strange, R., Mitrenga, T., Wolfe, P., and Kaeck, M. 2000. Fibronectin fragments induce MMP activity in mouse mammary epithelial cells: Evidence for a role in mammary tissue remodeling. J. Cell Sci. 113: 795-806.

Schnaper, H.W., Grant, D.S., Stetler-Stevenson, W.G., Fridman, R., D'Orazi, G., Murphy, A.N., Bird, R.E., Hoythya, M., Fuerst, T.R., French, D.L., et al. 1993. Type IV collagenase(s) and TIMPs modulate endothelial cell morphogenesis in vitro. J. Cell. Physiol. 156: 235-246.

Schönbeck, U., Mach, F., and Libby, P. 1998. Generation of biologically active IL-1 beta by matrix metalloproteinases: A novel caspase-1-independent pathway of IL-1 beta processing. J. Immunol. 161: 3340-3346.

Senior, R.M. 1994. Matrilysin is much more efficient than other matrix metalloproteinases in the proteolytic inactivation of alpha 1-antitrypsin. Biochem. Biophys. Res. Comm. 204: 613-620.

Sires, U.I., Murphy, G., Baragi, V.M., Fliszar, C.J., Welgus, H.G., Sternlicht, M.D., Lochter, A., Sympson, C.J., Huey, B., Rougier, J.P., et al. 1999. The stromal proteinase MMP3/stromelysin-1 promotes mammary carcinogenesis. Cell 98: 137146.

Sternlicht, M., Coussens, L.M., Vu, T.H., and Werb, Z. 2000. Biology and regulation of the matrix metalloproteinases. In Cancer drug discovery and development: Matrix metalloproteinase inhibitors in cancer therapy (ed. N.J. Clendeninn and K. Appelt), pp. 1-37. Humana Press Inc., Totowa, N.J.

Sympson, C.J., Talhouk, R.S., Alexander, C.M., Chin, J.R., Clift, S.M., Bissell, M.J., and Werb, Z. 1994. Targeted expression of stromelysin-1 in mammary gland provides evidence for a role of proteinases in branching morphogenesis and the requirement for an intact basement membrane for tissue-specific gene expression. J. Cell Biol. 125: 681-693.

Thrailkill, K.M., Quarles, L.D., Nagase, H., Suzuki, K., Serra, D.M., and Fowlkes, J.L. 1995. Characterization of insulinlike growth factor-binding protein 5-degrading proteases produced throughout murine osteoblast differentiation. Endocrinology 136: 3527-3533.

Ugwu, F., Van Hoef, B., Bini, A., Collen, D., and Lijnen, H.R.
1998. Proteolytic cleavage of urokinase-type plasminogen activator by stromelysin-1 (MMP-3). Biochemistry 37: 7231-7236.

Uzui, H., Lee, J., Shimizu, H., Tsutani, H., and Ueda, T. 2000. The role of protein-tyrosine phosphorylation and gelatinase production in the migration and proliferation of smooth muscle cells. Atherosclerosis 149: 51-59.

Vu, T.H., Shipley, J.M., Bergers, G., Berger, J.E., Helms, J.A., Hanahan, D., Shapiro, S.D., Senior, R.M., and Werb, Z. 1998. MMP-9/gelatinase B is a key regulator of growth plate angiogenesis and apoptosis of hypertrophic chondrocytes. Cell 93: 411-422.

Whitelock, J.M., Murdoch, A.D., Iozzo, R.V., and Underwood, P.A. 1996. The degradation of human endothelial cell-derived perlecan and release of bound basic fibroblast growth factor by stromelysin, collagenase, plasmin, and heparanases. J. Biol. Chem. 271: 10079-10086.

Witty, J.P., Wright, J.H., and Matrisian, L.M. 1995. Matrix metalloproteinases are expressed during ductal and alveolar mammary morphogenesis, and misregulation of stromelysin-1 in transgenic mice induces unscheduled alveolar development. Mol. Biol. Cell 6: 1287-1303.

Wu, H.B., Lee, C.Y., and Rechler, M.M. 1999. Proteolysis of insulin-like growth factor binding protein-3 in serum from pregnant, non-pregnant and fetal rats by matrix metalloproteinases and serine proteases. Hormone Metab. Res. 31: 186-191.

Yamamoto, H., Flannery, M.L., Kupriyanov, S., Pearce, J., McKercher, S.R., Henkel, G.W., Maki, R.A., Werb, Z., and Oshima, R.G. 1998. Defective trophoblast function in mice with a targeted mutation of Ets2. Genes \& Dev. 12: 13151326.

Yu, Q. and Stamenkovic, I. 2000. Cell surface-localized matrix metalloproteinase-9 proteolytically activates TGF-beta and promotes tumor invasion and angiogenesis. Genes \& Dev. 14: $163-176$. 


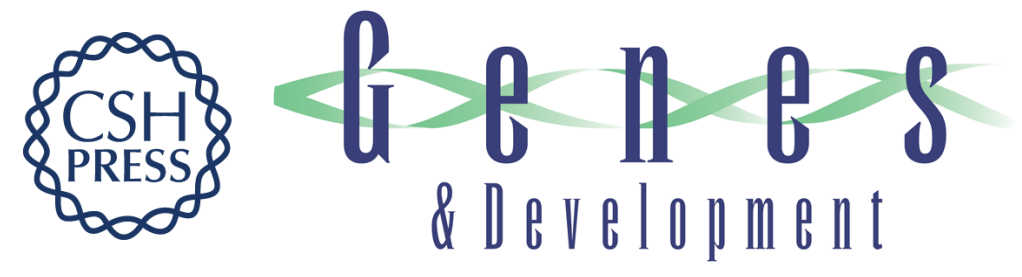

\title{
Matrix metalloproteinases: effectors of development and normal physiology
}

\author{
Thiennu H. Vu and Zena Werb
}

Genes Dev. 2000, 14:

Access the most recent version at doi:10.1101/gad.815400

$\begin{array}{ll}\text { References } & \begin{array}{l}\text { This article cites } 73 \text { articles, } 34 \text { of which can be accessed free at: } \\ \text { http://genesdev.cshlp.org/content/14/17/2123.full.html\#ref-list-1 }\end{array}\end{array}$

License

Email Alerting

Receive free email alerts when new articles cite this article - sign up in the box at the top Service right corner of the article or click here.

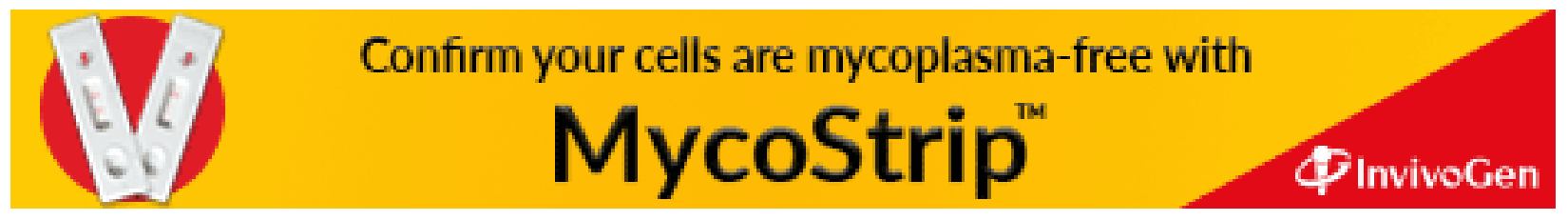

